\title{
Effort-Reward Imbalance, Resilience and Perceived Organizational Support: A Moderated Mediation Model of Fatigue in Chinese Nurses
}

This article was published in the following Dove Press journal: Risk Management and Healthcare Policy

\author{
Li Liu (D) \\ $\mathrm{Di} \mathrm{Wu}^{2}$ \\ Lulu Wang' \\ Yunting Qu' \\ Hui Wu' \\ 'Department of Social Medicine, School \\ of Public Health, China Medical \\ University, Shenyang, Liaoning, People's \\ Republic of China; ${ }^{2}$ School of \\ Fundamental Sciences, China Medical \\ University, Shenyang, Liaoning, People's \\ Republic of China
}

Correspondence: Hui Wu

Department of Social Medicine, School of Public Health, China Medical University,

No. 77 Puhe Road, Shenyang North New Area, Shenyang II0122, People's Republic of China

Tel +861890091 0568

Email hwu@cmu.edu.cn
Purpose: Fatigue undermines nurses' well-being and work performance worldwide. Work stress is a critical factor that causes nurses' fatigue, but the solutions are not well known. This study aimed to identify the moderated mediation model of fatigue involving effortreward imbalance (ERI), resilience and perceived organizational support (POS) in Chinese nurses.

Methods: In a cross-sectional investigation conducted in Liaoning Province of China, 996 nurses completed the self-administered measurements of fatigue, ERI, resilience and POS. Moderated mediation was tested using the PROCESS procedure. The "pick-a-point" method was adopted to assess conditional effect on fatigue.

Results: The indirect effect of ERI on fatigue through resilience was significant $(\mathrm{a} \times \mathrm{b}=$ 0.119, $\mathrm{BCa} 95 \% \mathrm{CI}: 0.094$ to 0.146 ), indicating a partial mediation of resilience. POS moderated the association of resilience with fatigue $(\beta=-0.056, p=0.006)$. The higher the level of POS was, the stronger the resilience-fatigue association became. Thus, the mediating role of resilience in the ERI-fatigue association was gradually increased with the increase of POS level (low POS: 0.073; moderate POS: 0.095; high POS: 0.116).

Conclusion: Resilience could partially mediate the ERI-fatigue association. POS could act as a moderator that enhanced the association of resilience with fatigue. A moderated mediation model of fatigue was demonstrated in Chinese nurses. Managers should establish a balanced social reciprocity, and improve nurses' resilience and POS in order to decrease nurses' fatigue.

Keywords: fatigue, effort-reward imbalance, resilience, perceived organizational support, nurses

\section{Introduction}

Fatigue generally comprises a series of physical and mental symptoms that result from responses to excessive demands without adequate overall recovery. ${ }^{1}$ Fatigue can reduce employee's work performance, even increase human errors and accidents in the workplace. ${ }^{2,3}$ Moreover, for occupational populations, fatigue is related to certain health problems, such as depression and musculoskeletal disorders. ${ }^{3-5}$ Healthcare workers, especially nurses, have to routinely confront the physical and psychological challenges of fatigue that can impair their overall health and work performance around the world. ${ }^{6-8}$ At the same time, reduced work efficiency and quality caused by fatigue can directly undermine patient satisfaction and safety of nursing services. ${ }^{9,10}$ Thus, from the perspective of nursing management, individual 
and organizational factors related to fatigue should be given more attention to develop the prevention and treatment strategies in nurses.

Nurses in China, in particular, frequently suffer from fatigue. ${ }^{7,11}$ For a long time, there are many stress factors in the nursing work settings, including demand overload, role conflict, shift rotation, failed social reciprocity, ${ }^{7,11,12}$ which make them susceptible to both physical and mental fatigues. Sufficient evidence has indicated that work stress is a vital predictor of fatigue in nurses. ${ }^{7,13}$ In China, nurses' work stress has been increasing due to the shortage of nursing professionals and the increased demand from patients for high-quality nursing service. ${ }^{7,14}$ As a classical model of work stress, the Effort Reward Imbalance (ERI) model was proposed by Siegrist based on reciprocity principle. ${ }^{15}$ The ERI model asserts that work with imbalanced extrinsic effort and reward signifies a failed social reciprocity that elicits work stress. There is increasing evidence that ERI has a positive association with fatigue across occupational groups, including nurses. ${ }^{7,16}$ Because the ERI model directly focuses on employee's interests, it has an innate advantage in measuring the level of work stress among occupational groups. ${ }^{17}$ Furthermore, based on previous findings, ${ }^{7,18,19}$ work stress also could indirectly cause fatigue by changing some specific psychological states in nurses.

Based on the conservation of resource (COR) theory, ${ }^{20}$ effective coping with work stress can be realized by using both internal and external resources. In recent years, internal coping resources have been focused on positive psychological capacity, while organizational support acts as an external coping resource in workplaces. ${ }^{17,19}$ As a research focus of positive psychology, resilience refers to a kind of positive capacity to pursue career success through bouncing back from difficulties and failures, even going beyond them. ${ }^{21}$ As a result, resilience can promote employees to utilize various positive resources to bring about many good outcomes in the workplace. ${ }^{22-}$ ${ }^{24}$ Resilience has significantly positive effects on the workrelated outcomes and well-being of nurses. ${ }^{23-25}$ Prior evidence has suggested that resilience is negatively related to fatigue across occupational populations. ${ }^{26,27}$ In addition, resilience has a partial mediation in the association of empathy with work engagement among hemodialysis nurses in China. ${ }^{22}$ Liu et al reported that resilience played a mediating role in the relation between nursing occupational risk and job performance. ${ }^{28}$ Also, resilience showed a partial mediation in the associations of emotional exhaustion and depersonalization with psychological distress among Chinese female nurses. ${ }^{29}$ Although some work stress factors influence the resilience level of nurses, ${ }^{30,31}$ the mediation effect of resilience in the ERIfatigue relation has not been reported among nurses to our best knowledge.

Perceived organizational support (POS) refers to employees' perception about help, affirmation and concern from their organizations. ${ }^{32}$ According to theoretical and empirical evidence, POS increases employee's positive work attitude, commitment and engagement, and can bring about a series of positive effects on employees' performance and well-being in the workplace. ${ }^{19,33}$ Moreover, POS acts as an effectively contextual resource to influence the effects of work stress and psychological capacities on work-related outcomes. ${ }^{27,34,35}$ Thus, the moderating role of POS has been given an increasing attention. Lartey et al reported the moderating role of POS in the relation between emotional labor and work attitudes among health professionals. ${ }^{35}$ Among Chinese doctors, Hao et al revealed that POS played a positive moderating role on the association between work-family conflict and depressive symptoms, ${ }^{34}$ and Qiu et al also indicated a moderation effect of POS on the associations of resilience with physical and mental fatigues. ${ }^{27}$ Accordingly, POS could act as a moderator on the relations of work stress and resilience with fatigue in Chinese nurses.

In light of the conceptual frameworks and practical concerns, a moderated mediation model of fatigue involving ERI, resilience and POS in nurses has not been examined to our best knowledge. Once this model is confirmed, it may contribute both theoretically and practically to the precise management of nurse fatigue from the perspective of integration of internal and external resources. The present study aimed to examine two hypotheses in Chinese nurses: (1) Resilience could mediate the association between ERI and fatigue, and (2) POS could moderate the direct association between ERI and fatigue, and the indirect association between ERI and fatigue through resilience.

\section{Materials and Methods Study Design and Sample}

A cross-sectional survey was carried out during April to July 2018 in Liaoning Province, China. Multi-stage stratified sampling method was adopted. Firstly, the province 
was geographically divided into five regions: eastern, western, southern, northern and central regions. Then, two tertiary hospitals were randomly sampled in each region. In China, the tertiary hospital is the highest level of general hospital and possesses more than 500 beds. In total, ten tertiary hospitals were sampled. The minimum sample size was calculated using the formula: $\mathrm{n}=\left(Z_{\alpha / 2}\right)^{2} P(1-P) /$ $\delta^{2}$. In the formula, $\alpha=0.05, Z_{\alpha / 2}=1.96 . P$ is the estimated prevalence of fatigue symptoms in nurses, which is estimated according to the results of previous studies and trial test. In this study, $P=0.25$. $\delta$ is tolerable error, and $\delta=$ 0.04 in this study. The design effect (deff) of the study was 2. Thus, we identified 900 as the minimum sample size for this study. During the calculation, the response rate was estimated to be 0.80 . Finally, 1120 nurses with a length of service $\geq 1$ year were sampled from different departments in these selected hospitals. Thus, 112 nurses were equally and randomly chosen from each selected hospital. Given the differences in the size and number of departments among these selected hospitals, the departments of the participants were divided into several categories: internal medicine, surgery, obstetrics and gynecology, pediatrics, and others. In this study, the nurses were not equally chosen from each department. Random number table method was used to complete random sampling. Participants were asked to complete a set of selfadministered questionnaires after their scheduled shifts in a private place. There was no interference in the process, and it took about 10 minutes for the participants to complete the questionnaires. Questionnaires with missing data for any item were excluded from the final analysis. In this study, 996 (88.9\%) effective responses were obtained. The study was conducted in accordance with the Declaration of Helsinki, and the protocol was approved by the Committee on Human Experimentation of China Medical University. All of the participants voluntarily and anonymously completed the survey, and their written informed consents were obtained.

\section{Measurements}

The level of fatigue was assessed using the Chinese version of the Chalder Fatigue Scale (CFS). ${ }^{36}$ The CFS has 14 items under two dimensions: physical fatigue (8 items) and mental fatigue (6 items). A dichotomized response is designed for each item: 0 (no symptom) and 1 (have symptom). The summed score of the CFS ranges from 0 to14. The severity of fatigue increases with the increase of the CFS score. The CFS has been used among Chinese medical personnel. ${ }^{27,37,38}$ The Cronbach's $\alpha$ coefficient of the CFS was 0.86 in this study.

The extrinsic effort and reward subscales of the Chinese version of the ERI scale were adopted in this study. ${ }^{15,39}$ The extrinsic effort subscale consists of 6 items, while the reward subscale consists of 11 items. Each item is scored on a 5-point Likert-type scale. Firstly, respondents need to express their attitudes toward the statements about working situations: 1 (disagree) or 5 (agree). Secondly, respondents need to assess the extent to which they feel distressed: from 2 (not distressed) to 5 (very distressed) or from 4 (not distressed) to 1 (very distressed). The summed scores of extrinsic effort and reward are calculated, respectively. In this study, work stress was indicated by ERI. The computing formula of ERI is extrinsic effort/(reward $\times 0.5454)$. A higher ERI value indicates a higher level of work stress. The ERI scale has been widely applied among Chinese healthcare workers. ${ }^{7,17,19}$ In our sample, the Cronbach's $\alpha$ coefficients of the extrinsic effort and reward subscales were 0.84 and 0.94 , respectively.

The Chinese version of Connor-Davidson Resilience Scale (CD-RISC) was used to assess the level of resilience. ${ }^{40,41}$ The scale consists of 25 items that describe the personal perceptions about resilience. Each item is scored on a 5-point Likert-type scale ranging from 0 (not true at all) to 4 (true all the time). A total score of resilience is summed ranging from 0 to 100 . The higher the CD-RISC score, the higher the level of resilience. The CD-RISC scale has been extensively used among Chinese nurses. ${ }^{27,42}$ The Cronbach' $\alpha$ coefficient of the scale was 0.97 in this study.

The level of POS was measured using the Chinese version of the Survey of Perceived Organizational Support (SPOS). ${ }^{43}$ The scale consists of 9 items that describe the perceptions about organization's valuation and caring about employees. Each item has a 7-point Likert-type scale ranging from 1 (strongly disagree) to 7 (strongly agree). A total score of SPOS is summed ranging from 9 to 63. Higher SPOS scores indicate higher POS levels. The SPOS has been used across occupational populations in China. ${ }^{19,27,34}$ In our sample, the Cronbach's $\alpha$ coefficient of the scale was 0.91 .

Demographics including age (yrs), gender, marital status and education were collected. Marital status was divided into two groups: single/divorced/widowed/separated and married/cohabited. Education was divided into two groups: junior college or lower and bachelor college 
or higher. Some working characteristics were collected. Job rank had two categories: staff and director. Monthly income (RMB, yuan) was divided into four groups including <3000 yuan, 3000-4999 yuan, 5000-8000 yuan and $>8000$ yuan. Weekly work time (hrs) had two categories: $\leq 40 \mathrm{hrs}$ and $>40 \mathrm{hrs}$. Also, night shift information was collected.

\section{Statistical Analysis}

Number (n), percentage (\%), mean and standard deviation (SD) were adopted to describe the study variables appropriately. Comparison of the level of fatigue was conducted using Student's $t$-test or one-way ANOVA. Correlations among continuous variables were assessed by calculating Pearson's correlation coefficients. The moderated mediation was examined using the PROCESS procedure with centered variables. ${ }^{44}$ In the regression analyses, parameter estimation was performed based on 5000 bootstrapped samples. When a bias corrected and accelerated $95 \%$ confidence interval (BCa 95\% CI) does not contain zero, the mediating role is statistically significant. The "picka-point" method was used to estimate conditional effect (simple slopes) on fatigue. Statistical analysis software was the SPSS 21.0 (IBM, Asia Analytics Shanghai). A two-tailed $p<0.05$ was considered statistically significant.

\section{Results}

\section{Characteristics of Subjects}

The demographics and working characteristics of subjects and comparisons of fatigue are displayed in Table 1. Of these subjects, gender, marital status and education were not significantly related to the level of fatigue. In terms of working characteristics, $650 \quad(65.3 \%)$ subjects had

Table I Demographics and Working Characteristics of Subjects and Comparisons of Fatigue

\begin{tabular}{|c|c|c|c|c|c|c|}
\hline Variables & $\mathbf{n}$ & $\%$ & Mean & SD & $\mathbf{F} / \mathbf{t}$ & $\mathbf{p}$ \\
\hline \multicolumn{7}{|l|}{ Demographics } \\
\hline Gender & & & & & $1.677^{\mathrm{a}}$ & 0.095 \\
\hline Men & 115 & 11.5 & 7.46 & 3.14 & & \\
\hline Women & 881 & 88.5 & 6.92 & 4.19 & & \\
\hline Marital status & & & & & $1.221^{\mathrm{a}}$ & 0.222 \\
\hline Single/divorced/widowed/separated & 387 & 38.9 & 7.17 & 3.86 & & \\
\hline Married/cohabited & 609 & 61.1 & 6.86 & 4.22 & & \\
\hline Education & & & & & $0.47 I^{\mathrm{a}}$ & 0.638 \\
\hline Junior college or lower & 232 & 23.3 & 6.88 & 3.72 & & \\
\hline Bachelor college or higher & 764 & 76.7 & 7.01 & 4.19 & & \\
\hline \multicolumn{7}{|l|}{ Working characteristics } \\
\hline Job rank & & & & & $1.56 \mathrm{I}^{\mathrm{a}}$ & 0.121 \\
\hline Staff & 896 & 90.0 & 6.93 & 4.18 & & \\
\hline Director & 100 & 10.0 & 7.45 & 3.05 & & \\
\hline Monthly income (RMB, yuan) & & & & & $1.090^{\mathrm{b}}$ & 0.352 \\
\hline$<3000$ & 162 & 16.3 & 7.09 & 3.27 & & \\
\hline $3000-4999$ & 293 & 29.4 & 6.67 & 3.46 & & \\
\hline $5000-8000$ & 278 & 27.9 & 7.28 & 4.74 & & \\
\hline$>8000$ & 263 & 26.4 & 6.94 & 4.41 & & \\
\hline Weekly working time (hrs) & & & & & $12.866^{\mathrm{a}}$ & $<0.001$ \\
\hline$\leq 40$ & 346 & 34.7 & 4.84 & 3.91 & & \\
\hline$>40$ & 650 & 65.3 & 8.12 & 3.70 & & \\
\hline Night shift & & & & & $2.26 \mathrm{I}^{\mathrm{a}}$ & 0.024 \\
\hline Yes & 718 & 72.1 & 7.17 & 3.98 & & \\
\hline No & 278 & 27.9 & 6.49 & 4.31 & & \\
\hline
\end{tabular}

Notes: ${ }^{a} t$ value; ${ }^{b} F$ value.

Abbreviation: SD, standard deviation. 
a weekly working time $>40 \mathrm{hrs}$, and they reported a higher fatigue level $(t=12.866, p<0.001)$ than those with a weekly working time $\leq 40 \mathrm{hrs}$. Nurses with night shift $(718,72.1 \%)$ had a higher fatigue level compared with those without night shift $(t=2.261, p=0.024)$. However, job rank and monthly income were not significantly related to the fatigue of nurses.

\section{Correlations Among ERI, Resilience, POS and Fatigue}

The correlations among continuous variables are shown in Table 2. In our sample, the mean of age was $32.65 \mathrm{yrs}$ (SD =7.35). The mean of fatigue score was $6.98(\mathrm{SD}=4.08)$. ERI was positively correlated with fatigue $(r=0.517, p<$ $0.001)$, whereas it was negatively correlated with resilience $(r=-0.368, p<0.001)$. Both resilience $(r=-0.543$, $p<0.001)$ and POS $(r=-0.231, p<0.001)$ were negatively correlated with fatigue.

\section{Mediating Role of Resilience}

The total effect of ERI on fatigue was positively significant $(\beta=0.492, p<0.001)$. ERI showed a significantly indirect effect on fatigue through resilience $(\mathrm{a} \times \mathrm{b}=0.119$, BCa 95\% CI: 0.094 to 0.146 ), which indicated a partial mediation of resilience. The mediation model of resilience is depicted in Figure 1. Age, gender, weekly working time and night shift were adjusted. In addition, the proportion of resilience's mediation was $24.2 \%$ in the total effect of ERI on fatigue.

\section{Moderated Mediation}

POS only showed a moderating role in the association between resilience and fatigue $(\beta=-0.056, p=0.006)$, and this result is displayed in Figure 2. A simplified moderated mediation model was calculated and depicted in Figure 3. Simple slope analysis revealed that

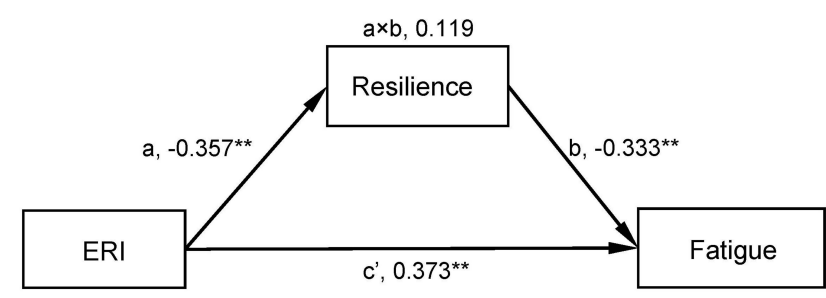

Figure I Mediation of resilience in the association between ERI and fatigue. Notes: Age, gender, weekly working time and night shift are adjusted. In order to simplify the model, these control variables are not shown. ERI is logarithmic. All path coefficients are $\beta$. ${ }^{*} p<0.00$ I (two-tailed).Abbreviation: ERI, effort-reward imbalance.

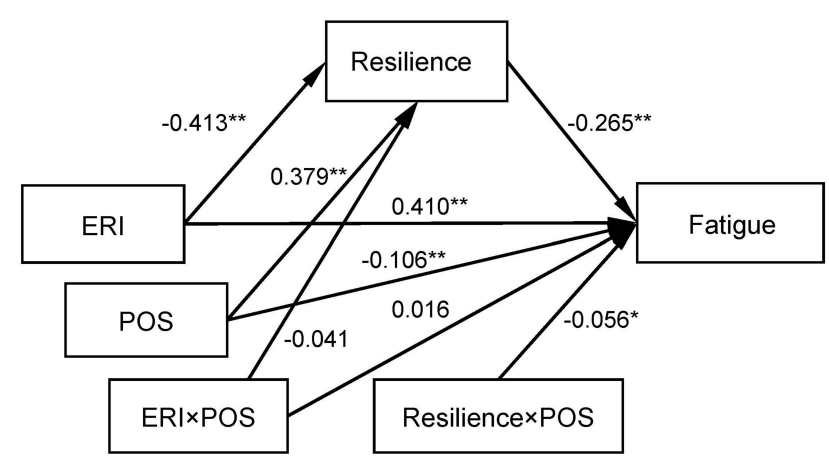

Figure 2 Full moderated mediation model predicting fatigue from ERI.Notes: Age, gender, weekly working time and night shift are adjusted. In order to simplify the model, these control variables are not shown. ERI is logarithmic. All path coefficients are $\beta$. ${ }_{p} p<0.01$; ${ }^{*} p<<0.001$ (two-tailed).Abbreviations: ERI, effort-reward imbalance; POS, perceived organizational support.

resilience was negatively associated with fatigue in low POS $(\beta=-0.205, p<0.001)$, moderate POS $(\beta=-0.265, p<0.001)$, and high POS $(\beta=-0.325$, $p<0.001)$ groups. We plotted the moderation of POS (1 SD below the mean, mean $\pm 1 \mathrm{SD}$, and $1 \mathrm{SD}$ above the mean, respectively) in Figure 4. As shown in Table 3, the mediating role of resilience in the ERI-fatigue association was gradually increased with the increase of POS level (low POS: 0.073, BCa 95\% CI: 0.044 to

Table 2 Correlations Among Continuous Variables

\begin{tabular}{|c|c|c|c|c|c|c|}
\hline \multirow[t]{2}{*}{ Variables } & \multirow[t]{2}{*}{ Mean } & \multirow[t]{2}{*}{ SD } & \multicolumn{4}{|c|}{ Correlations (r) } \\
\hline & & & $\mathbf{I}$ & 2 & 3 & 4 \\
\hline I. Age (yrs) & 32.65 & 7.35 & - & & & \\
\hline 2. ERI & 1.01 & 0.14 & 0.049 & - & & \\
\hline 3. Resilience & 57.17 & 23.90 & -0.020 & $-0.368 * *$ & - & \\
\hline 4. POS & 39.07 & 9.81 & -0.028 & $0.120 * *$ & $0.379 * *$ & - \\
\hline 5. Fatigue & 6.98 & 4.08 & 0.026 & $0.517^{* *}$ & $-0.543 * *$ & $-0.231 * *$ \\
\hline
\end{tabular}

Notes: The mean of ERI is logarithmic. ${ }^{*} p<<0.001$ (two-tailed).

Abbreviations: SD, standard deviation; ERI, effort-reward imbalance; POS, perceived organizational support. 


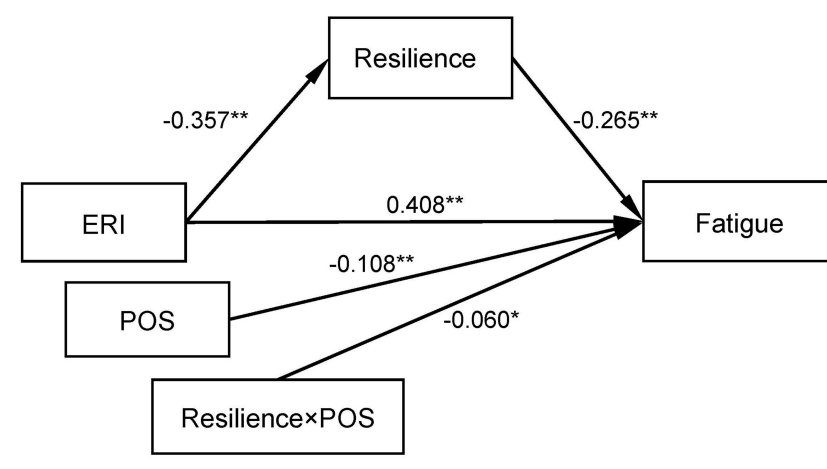

Figure 3 Simplified moderated mediation model predicting fatigue from ERI. Notes: Age, gender, weekly working time and night shift are adjusted. In order to simplify the model, these control variables are not shown. ERI is logarithmic. All path coefficients are $\beta .{ }^{*} p<0.01$; ${ }^{*} p<<0.001$ (two-tailed).Abbreviations: ERI, effort-reward imbalance; POS, perceived organizational support.

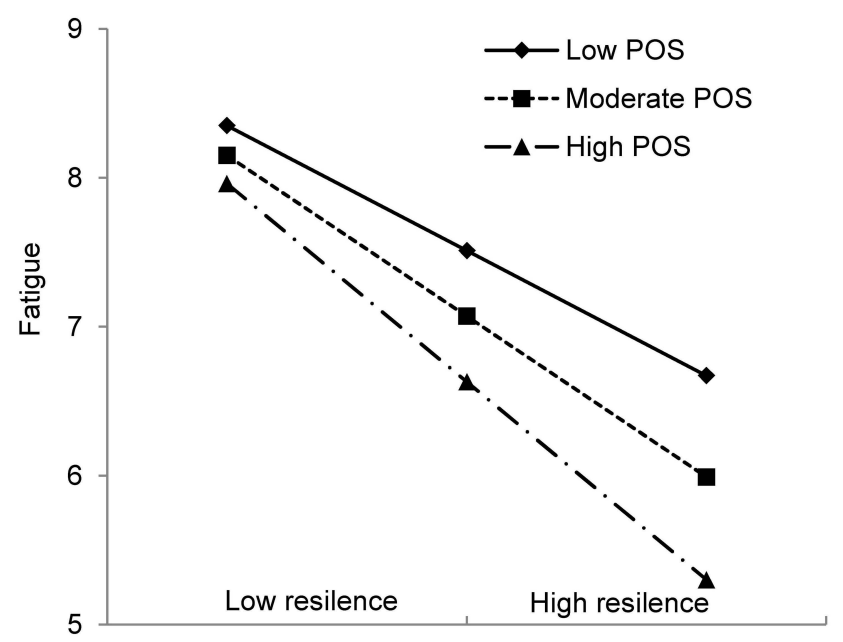

Figure 4 Moderation of POS on the resilience-fatigue association.Abbreviation: POS, perceived organizational support.

0.103; moderate POS: 0.095 , BCa 95\% CI: 0.068 to 0.122; high POS: $0.116, \mathrm{BCa} 95 \% \mathrm{CI}$ : 0.087 to 0.148 ).

\section{Discussion}

In the present study, the mean level of fatigue was $6.98 \pm$ 4.08 in Chinese nurses, which was lower than the levels of fatigue from previous studies in China. ${ }^{37,45}$ Also, comparison of the fatigue between nurses and other occupational populations with high work stress is helpful to understand the situation of nurses' fatigue across groups. The level of fatigue in our nurse sample was lower than the levels of Chinese doctors $(8.02 \pm 3.39)$ and scientific and technical personnel $(7.28 \pm 3.37) .{ }^{38,46}$ However, it was roughly consistent with the fatigue of Chinese doctors in a recent study $(6.91 \pm 3.19) .{ }^{27}$ In the past two decades, nurses in
Table 3 Conditional Indirect Effects of ERI on Fatigue Through Resilience at Values of POS

\begin{tabular}{|l|l|l|}
\hline \multirow{2}{*}{ POS } & ERI & \\
\cline { 2 - 3 } & Effect & $\mathbf{9 5 \%} \mathbf{~ C I}$ \\
\hline Low (I SD below the mean) & 0.073 & $0.044,0.103$ \\
Moderate (mean \pm I SD) & 0.095 & $0.068,0.122$ \\
High (I SD above the mean) & 0.116 & $0.087,0.148$ \\
\hline
\end{tabular}

Notes: Age, gender, weekly working time and night shift are adjusted. ERI is logarithmic.

Abbreviations: ERI, effort-reward imbalance; POS, perceived organizational support; SD, standard deviation; $\mathrm{Cl}$, confidence interval.

China have been facing serious fatigue problem caused by the relative shortage of human resources. ${ }^{7,11,47}$ In particular, the fatigue level of nurses in large general hospitals is considered to be higher than that in primary hospitals and private hospitals. ${ }^{37}$ With the development of social economy and the progress of health care system reform, new changes have taken place in nursing service in China. ${ }^{48}$ The new health care system reforms have been gradually implemented, and the current measures mainly include increasing medical service fees, cancelling outpatient infusion, improving hierarchical medical system and encouraging multi-point practice. Furthermore, the application of new technology and equipment and the increase of male nurses' proportion are conducive to alleviating the fatigue of nurses. Future retrospective or prospective longitudinal studies need to be carried out to confirm these inferences.

The results of this study firstly indicated a moderated mediation model of fatigue involving ERI, resilience and POS in Chinese nurses. POS acted as a moderator for the direct effect of resilience on fatigue and the mediating role of resilience in the association between ERI and fatigue. In consistent with previous findings, ${ }^{7,16}$ we found that ERI had a positive association with the level of Chinese nurses' fatigue. Therefore, work stress reduction should be included in the interventions of fatigue. Nursing managers should rationalize the work demand (such as workload, overtime and responsibility) and work reward (such as fairness, promotion, stability, respect, support and income) of nurses to balance extrinsic effort and reward at work. The new medical reform measures mentioned above could help to gradually reduce the work stress of medical staff in China.

In addition to the direct effect of ERI, ERI also could indirectly result in fatigue through reducing the level of resilience in this study. Our result indicated that resilience 
could be considered a psychological resource to deal with fatigue in Chinese nurses. When nurses have a high level of resilience, they tend to possess more energy and proactivity to regulate their emotions and perceptions. ${ }^{49}$ As a state positive psychological capability, resilience can be developed. For instance, a previous study confirmed that resilience training improved the level of resilience, and decreased the level of occupational stress in nurses from intensive care units. $^{50}$ Another study showed that a participatory action research-based resilience enhancement project helped student nurses improve resilience through enhancing their knowledge and skills in nursing theory and practice, as well as positive thinking and behavior during clinical practices. ${ }^{51}$ Therefore, to alleviate fatigue and other negative outcomes of work stress, evidence-based resilience improvement programs should be carried out in the practice of nursing management.

In addition to resilience, POS acted as a contextual resource for coping with fatigue in Chinese nurses. To be specific, POS not only exerted a direct effect on fatigue, but also moderated the association of resilience with fatigue. ${ }^{27}$ The results of simple slope analyses indicated that the resilience-fatigue association was gradually increased with the increase of POS level. There are synergistic effects among positive resources. That means one resource can trigger another resource, or increase the effect size of the latter. Thus, the synergy could be an explanation for the moderation of POS on resilience' effect. ${ }^{27,35,49}$ In fact, it has been widely confirmed that POS can play a moderating role on the work-related effects of psychological resources, including many studies in medical staff. $^{27,35}$ Nursing managers should provide supportive settings for nurses to improve their POS in workplaces. Due to the moderation of POS on the resilience-fatigue association, the conditional indirect effect of ERI on fatigue through resilience was found based on the moderated mediation model of this study.

The study can have two practical implications for nursing management. Firstly, resilience acted as a partial mediator of the association between ERI and nurses' fatigue. Based on this finding, one suggestion is that a balanced reciprocity relationship should be built and maintained, which will help nurses develop psychological resilience to reduce fatigue. Secondly, the moderated mediation model demonstrated that POS functioned as an important contextual resource to enhance the direct effect of resilience on fatigue and the mediating role of resilience. The findings showed that resilience could more effectively reduce fatigue for nurses with adequate POS. Based on this finding, another suggestion is that nursing managers should improve nurses' POS by providing supportive settings, including giving adequate help, affirming their contributions and caring about their well-being.

Several limitations of this study must be acknowledged. First of all, the hypotheses of this study were based on theoretical and empirical evidence, but the causal relations of ERI, resilience and POS with fatigue could not be concluded due to the cross-sectional survey design. These causal relations need to be verified in future longitudinal studies. Secondly, this study was only carried out in the tertiary hospitals of a province in Northeast China, and the representativeness of our sample could be reduced. Further studies should be conducted to verify our findings in different levels and regions of hospital nurses. Thirdly, there are many confounding factors that are related to the study variables, such as depression and anxiety. However, in this study, we did not control the potential impacts of these factors. We only regarded "with a length of service $\geq 1$ year" as the inclusion and exclusion criteria. A rigorous study design is needed to control confounding factors in future studies. The last but not the least, we only used selfreport questionnaires to measure the psychosocial variables in this study, which could be subjected to recall and reporting bias. Although anonymity and quality control procedure are conducive to reducing common method bias, panel data obtained from repeated surveys can reduce the bias.

\section{Conclusion}

In Chinese nurses, resilience could partially mediate the effect of ERI on fatigue. POS could act as a moderator that enhanced the association of resilience with fatigue. Our findings demonstrated a moderated mediation model of fatigue involving ERI, resilience and POS and yielded practical implications for the coping strategies of fatigue in Chinese nurses. Managers should establish a balanced social reciprocity, and improve nurses' resilience and POS in order to decrease nurses' fatigue.

\section{Funding}

This study was supported by the National Natural Science Foundation of China (No. 71673300, Hui Wu). The authors would like to thank all of the nurses who voluntarily participated in this study. 


\section{Disclosure}

The authors report no conflicts of interest in this work.

\section{References}

1. Aaronson LS, Teel CS, Cassmeyer V, et al. Defining and measuring fatigue. Image J Nurs Sch. 1999;31(1):45-50. doi:10.1111/j.15475069.1999.tb00420.x

2. Caldwell JA, Caldwell JL, Thompson LA, Lieberman HR. Fatigue and its management in the workplace. Neurosci Biobehav Rev. 2019;96:272-289. doi:10.1016/j.neubiorev.2018.10.024

3. Lock AM, Bonetti DL, Campbell ADK. The psychological and physiological health effects of fatigue. Occup Med (Lond). 2018;68 (8):502-511. doi:10.1093/occmed/kqy109

4. Rose DM, Seidler A, Nübling M, et al. Associations of fatigue to work-related stress, mental and physical health in an employed community sample. BMC Psychiatry. 2017;17(1):167. doi:10.1186/ s12888-017-1237-y

5. Younan L, Clinton M, Fares S, Jardali FE, Samaha H. The relationship between work-related musculoskeletal disorders, chronic occupational fatigue, and work organization: a multi-hospital cross-sectional study. $J$ Adv Nurs. 2019;75(8):1667-1677. doi:10.1111/jan.13952

6. Austin S, Fernet C, Trépanier SG, Lavoie-Tremblay M. Fatigue in new registered nurses: a 12-month cross-lagged analysis of its association with work motivation, engagement, sickness absence, and turnover intention. J Nurs Manag. 2020;28(3):606-614. doi:10. 1111/jonm. 12962

7. Huang H, Liu L, Yang S, Cui X, Zhang J, Wu H. Effects of job conditions, occupational stress, and emotional intelligence on chronic fatigue among Chinese nurses: a cross-sectional study. Psychol Res Behav Manag. 2019;12:351-360. doi:10.2147/PRBM.S207283

8. Johnston DW, Allan JL, Powell DJH, et al. Why does work cause fatigue? A real-time investigation of fatigue, and determinants of fatigue in nurses working 12-hour shifts. Ann Behav Med. 2019;53 (6):551-562. doi:10.1093/abm/kay065

9. Al Ma'mari Q, Sharour LA, Al Omari O. Fatigue, burnout, work environment, workload and perceived patient safety culture among critical care nurses. Br J Nurs. 2020;29(1):28-34. doi:10.12968/ bjon.2020.29.1.28

10. Samra HA, Smith BA. The effect of staff nurses' shift length and fatigue on patient safety and nurses' health: from the national association of neonatal nurses. Adv Neonatal Care. 2015;15(5):311. doi:10.1097/ANC.0000000000000230

11. Fang J, Qiu C, Xu H, You G. A model for predicting acute and chronic fatigue in Chinese nurses. $J$ Adv Nurs. 2013;69(3):546-558. doi:10.1111/j.1365-2648.2012.06029.x

12. McVicar A. Scoping the common antecedents of job stress and job satisfaction for nurses (2000-2013) using the job demands-resources model of stress. J Nurs Manag. 2016;24(2):E112-E136. doi:10.1111/ jonm. 12326

13. Jalilian H, Shouroki FK, Azmoon H, Rostamabadi A, Choobineh A. Relationship between job stress and fatigue based on job demand-control-support model in hospital nurses. Int J Prev Med. 2019;10(1):56. doi:10.4103/ijpvm.IJPVM_178_17

14. Yau SY, Xiao XY, Lee LY, Tsang AY, Wong SL, Wong KF. Job stress among nurses in China. Appl Nurs Res. 2012;25(1):60-64. doi:10.1016/j.apnr.2011.07.001

15. Siegrist J. Adverse health effects of high-effort/low-reward conditions. J Occup Health Psychol. 1996;1(1):27-41. doi:10.1037/ 1076-8998.1.1.27

16. Sembajwe G, Wahrendorf M, Siegrist J, et al. Effects of job strain on fatigue: cross-sectional and prospective views of the job content questionnaire and effort-reward imbalance in the GAZEL cohort. Occup Environ Med. 2012;69(6):377-384. doi:10.1136/oem.2010. 063503
17. Liu L, Chang Y, Fu J, Wang J, Wang L. The mediating role of psychological capital on the association between occupational stress and depressive symptoms among Chinese physicians: a cross-sectional study. BMC Public Health. 2012;12(1):219. doi:10.1186/1471-2458-12-219

18. Hong E, Lee YS. The mediating effect of emotional intelligence between emotional labour, job stress, burnout and nurses' turnover intention. Int J Nurs Pract. 2016;22(6):625-632. doi:10.1111/ijn.12493

19. Wang X, Liu L, Zou F, Hao J, Wu H. Associations of occupational stressors, perceived organizational support, and psychological capital with work engagement among Chinese female nurses. Biomed Res Int. 2017;2017:5284628.

20. Hobfoll SE. Conservation of resources. A new attempt at conceptualizing stress. Am Psychol. 1989;44(3):513-524. doi:10.1037/0003066X.44.3.513

21. Tugade MM, Fredrickson BL, Barrett LF. Psychological resilience and positive emotional granularity: examining the benefits of positive emotions on coping and health. J Pers. 2004;72(6):1161-1190. doi:10.1111/j.1467-6494.2004.00294.x

22. Cao X, Chen L. The impact of empathy on work engagement in hemodialysis nurses: the mediating role of resilience. Jpn J Nurs Sci. 2020;17(1):e12284. doi:10.1111/jjns.12284

23. Delgado C, Upton D, Ranse K, Furness T, Foster K. Nurses' resilience and the emotional labour of nursing work: an integrative review of empirical literature. Int J Nurs Stud. 2017;70:71-88. doi:10.1016/j.ijnurstu.2017.02.008

24. Walpita YN, Arambepola C. High resilience leads to better work performance in nurses: evidence from South Asia. J Nurs Manag. 2020;28(2):342-350. doi:10.1111/jonm.12930

25. Ang SY, Hemsworth D, Uthaman T, et al. Understanding the influence of resilience on psychological outcomes - comparing results from acute care nurses in Canada and Singapore. Appl Nurs Res. 2018;43:105-113. doi:10.1016/j.apnr.2018.07.007

26. Kitamura H, Shindo M, Tachibana A, Honma H, Someya T. Personality and resilience associated with perceived fatigue of local government employees responding to disasters. J Occup Health. 2013;55(1):1-5. doi:10.1539/joh.12-0095-BR

27. Qiu T, Yang Y, Liu C, et al. The association between resilience, perceived organizational support and fatigue among Chinese doctors: a cross-sectional study. J Affect Disord. 2020;265:85-90. doi:10.1016/j. jad.2020.01.056

28. Liu RH, Zou Y, Wang J, Lin Q, Wang F. Mediating effect of resilience on nursing occupational risk and job performance in nurses. Chin J Ind Hyg Occup Dis. 2019;37(8):580-584.

29. Zou G, Shen X, Tian X, et al. Correlates of psychological distress, burnout, and resilience among Chinese female nurses. Ind Health. 2016;54(5):389-395. doi:10.2486/indhealth.2015-0103

30. Öksüz E, Demiralp M, Mersin S, Tüzer H, Aksu M, Sarıkoc G. Resilience in nurses in terms of perceived social support, job satisfaction and certain variables. J Nurs Manag. 2019;27(2):423-432. doi:10.1111/jonm.12703

31. Ren Y, Zhou Y, Wang S, Luo T, Huang M, Zeng Y. Exploratory study on resilience and its influencing factors among hospital nurses in Guangzhou, China. Int J Nurs Sci. 2017;5(1):57-62. doi:10.1016/j.jjnss.2017.11.001

32. Eisenberger R, Armeli S, Rexwinkel B, Lynch PD, Rhoades L. Reciprocation of perceived organizational support. J Appl Psychol. 2001;86(1):42-51. doi:10.1037/0021-9010.86.1.42

33. Liu W, Zhao S, Shi L, et al. Workplace violence, job satisfaction, burnout, perceived organisational support and their effects on turnover intention among Chinese nurses in tertiary hospitals: a cross-sectional study. BMJ Open. 2018;8(6):e019525. doi:10.1136/ bmjopen-2017-019525

34. Hao J, Wang J, Liu L, Wu W, Wu H. Perceived organizational support impacts on the associations of work-family conflict or family-work conflict with depressive symptoms among Chinese doctors. Int J Environ Res Public Health. 2016;13(3):326. doi:10. 3390/ijerph 13030326 
35. Lartey JKS, Amponsah-Tawiah K, Osafo J. The moderating effect of perceived organizational support in the relationship between emotional labour and job attitudes: a study among health professionals. Nurs Open. 2019;6(3):990-997. doi:10.1002/nop2.295

36. Chalder T, Berelowitz G, Pawlikowska T, et al. Development of a fatigue scale. J Psychosom Res. 1993;37(2):147-153. doi:10.1016/ 0022-3999(93)90081-P

37. Liu TT, Du MS, Jia XP. Investigation and analysis on the fatigue of tertiary general hospital nurses in Anshan City. Mod Hosp Manag. 2014;12(1):46-48.

38. Liu L, Xu P, Zhou K, Xue J, Wu H. Mediating role of emotional labor in the association between emotional intelligence and fatigue among Chinese doctors: a cross-sectional study. BMC Public Health. 2018;18(1):881. doi:10.1186/s12889-018-5817-7

39. Yang WJ, Li J. Measurement of psychosocial factors in work environment: application of two models of occupational stress. Chin J Ind Hyg Occup Dis. 2004;22(6):422-426.

40. Connor KM, Davidson JR. Development of a new resilience scale: the connor-davidson resilience scale (CD-RISC). Depress Anxiety. 2003;18(2):76-82. doi:10.1002/da.10113

41. Yu X, Zhang J. Factor analysis and psychometric evaluation of the Connor-Davidson resilience scale (CD-RISC) with Chinese people. Soc Behav Pers. 2007;35(1):19-30. doi:10.2224/sbp.2007.35.1.19

42. Guo YF, Luo YH, Lam L, Cross W, Plummer V, Zhang JP. Burnout and its association with resilience in nurses: a cross-sectional study. J Clin Nurs. 2018;27(1-2):441-449. doi:10.1111/jocn.13952

43. Eisenberger R, Fasolo P, Davis-lamastro V. Perceived organizational support and employee diligence, commitment, and innovation. J Appl Psychol. 1990;75(1):51-59. doi:10.1037/0021-9010.75.1.51
44. Hayes AF. An index and test of linear moderated mediation. Multivariate Behav Res. 2015;50(1):1-22. doi:10.1080/00273171.20 14.962683

45. Liu WS, Tian Z, Wang XD, Gao YS. The investigation of working fatigue of clinical nurses. Mod Prev Med. 2011;38(10):1854-1856.

46. Yang T, Zhou D, Song M, Lan Y. Analysis of work-related fatigue characteristics and its influencing factors in scientific and technical personnel. Chin J Ind Hyg Occup Dis. 2015;33(2):100-103.

47. You LM, Ke YY, Zheng J, Wan LH. The development and issues of nursing education in China: a national data analysis. Nurse Educ Today. 2015;35(2):310-314. doi:10.1016/j.nedt.2014.10.004

48. Yip W, Fu H, Chen AT, et al. 10 years of health-care reform in China: progress and gaps in universal health coverage. Lancet. 2019;394 (10204):1192-1204. doi:10.1016/S0140-6736(19)32136-1

49. Mache S, Vitzthum K, Wanke E, David A, Klapp BF, Danzer G. Exploring the impact of resilience, self-efficacy, optimism and organizational resources on work engagement. Work. 2014;47 (4):491-500. doi:10.3233/WOR-131617

50. Babanataj R, Mazdarani S, Hesamzadeh A, Gorji MH, Cherati JY. Resilience training: effects on occupational stress and resilience of critical care nurses. Int J Nurs Pract. 2019;25(1):e12697. doi:10.1111/ijn.12697

51. Liang HF, Wu KM, Hung CC, Wang YH, Peng NH. Resilience enhancement among student nurses during clinical practices: a participatory action research study. Nurse Educ Today. 2019;75:22-27. doi:10.1016/j.nedt.2019.01.004
Risk Management and Healthcare Policy

\section{Publish your work in this journal}

Risk Management and Healthcare Policy is an international, peerreviewed, open access journal focusing on all aspects of public health, policy, and preventative measures to promote good health and improve morbidity and mortality in the population. The journal welcomes submitted papers covering original research, basic science, clinical \& epidemiological studies, reviews and evaluations, guidelines, expert opinion and commentary, case reports and extended reports. The manuscript management system is completely online and includes a very quick and fair peer-review system, which is all easy to use. Visit http://www.dovepress.com/testimonials.php to read real quotes from published authors. 